\title{
Wake Estimation and Optimal Control for Autonomous Aircraft in Formation Flight
}

\author{
Levi DeVries* and Derek A. Paley ${ }^{\dagger}$ \\ University of Maryland, College Park, MD, 20742, USA
}

\begin{abstract}
The continued development of sophisticated aircraft with high fidelity control systems will enable autonomous execution of challenging tasks such as aerial refueling and close formation flight. In order to achieve such tasks in autonomous flight, an aircraft must sense other aircraft in close proximity and position itself relative to them. For example, aerial refueling requires the follower aircraft to intercept the filling nozzle attached to the leader aircraft; also, formation-flying aircraft must position themselves strategically to realize benefits of aerodynamic efficiency. This paper uses lifting-line theory to represent a two-aircraft formation and presents a grid-based, recursive Bayesian filter for estimating the wake parameters of the leader aircraft using noisy pressure measurements distributed along the trailing aircraft's wing; the estimator also requires a binary, relative-altitude measurement to break the vertical symmetry. Optimal control strategies are presented to steer the follower aircraft to a desired position relative to the leader while simultaneously optimizing the observability of the leader's relative position. The control algorithms guide the follower aircraft along trajectories that maintain adequate observability, thereby guaranteeing estimator performance. Theoretical results are illustrated using numerical examples of two-aircraft formations.
\end{abstract}

\section{Nomenclature}

\section{$\mathcal{B}$ Body reference frame attached to follower aircraft}

\footnotetext{
*Graduate Student, Department of Aerospace Engineering, University of Maryland, and AIAA Student Member.

${ }^{\dagger}$ Associate Professor, Department of Aerospace Engineering and the Institute for Systems Research, University of Maryland, and AIAA Associate Fellow.
} 
$O \quad$ Origin of follower aircraft body frame

$\mathbf{b}_{i} \quad i^{\text {th }}$ basis vector of frame $\mathcal{B}, i=1, \ldots, 3$

$\mathbf{r}_{L} \quad$ Three-dimensional position of leader aircraft relative to follower

$\mathbf{r}$ Two-dimensional position of leader aircraft in the $\left(\mathbf{b}_{2}, \mathbf{b}_{3}\right)$ plane

$x_{L} \quad$ Position coordinate of leader aircraft relative to follower in the $\mathbf{b}_{1}$ direction

$y_{L} \quad$ Position coordinate of leader aircraft relative to follower in the $\mathbf{b}_{2}$ direction

$z_{L} \quad$ Position coordinate of leader aircraft relative to follower in the $\mathbf{b}_{3}$ direction

$\boldsymbol{V}_{f} \quad$ Velocity of follower aircraft with respect to leader aircraft

$V_{y} \quad$ Velocity of follower aircraft in the $\mathbf{b}_{2}$ direction

$V_{z} \quad$ Velocity of follower aircraft in the $\mathbf{b}_{3}$ direction

$b \quad$ Leader and follower aircraft wingspans

$\Gamma_{L} \quad$ Circulation strength of leader aircraft wake vortices

$w_{L} \quad$ Upwash wake created by leader aircraft

$\Phi \quad$ Flow potential function

$N \quad$ Number of horseshoe vortices in lifting-line model

$U_{\infty} \quad$ Freestream velocity

$\alpha \quad$ Angle of attack of freestream velocity

$\boldsymbol{n}_{i} \quad$ Unit vector normal to wing at wingspan location $y_{i}$

$w_{h s}$ Vertical component of flow generated by horseshoe vortices

$c \quad$ Chord length of follower aircraft wing

$\Delta C_{p}$ Differential pressure

$\Omega \quad$ Set of leader aircraft wake parameters

$M \quad$ Number of parameters characterizing the leader aircraft wake

$P \quad$ Number of measurement locations

$\phi \quad P \times 1$ column matrix of differential pressure measurements

$\xi \quad$ Unobservability index

$\lambda \quad$ Estimation condition number

$\sigma \quad$ Singular value of empirical observability gramian

$W_{O} \quad$ Empirical observability gramian

$\mathbf{e}_{i} \quad i^{\text {th }}$ unit vector in $\mathbb{R}^{M}, i=1, \ldots, M$

$\Sigma_{i} \quad$ Variance of Gaussian noise in $i^{\text {th }}$ sensor measurement

$\boldsymbol{\eta}_{i} \quad$ zero mean noise with variance $\Sigma_{i}$

$\theta \quad$ Binary relative-altitude measurement

$\boldsymbol{\mu} \quad(P+1) \times 1$ column matrix of measurements $(\phi, \theta)$

$\Psi \quad$ Motion matrix used in Bayesian filter

$J$ Trajectory optimization cost function

$T_{f} \quad$ Horizon time of optimal control calculation 
$T_{e} \quad$ Execution time of optimal control calculation

$K_{W F}$ constant gain of gradient descent control

\section{Introduction}

As airspaces of the world grow more crowded with manned and unmanned aircraft, closely coordinated formations may provide a method of organizing the sky. Applications of close formation flight have been shown to extend the range of individual platforms through aerial refueling $^{1,2}$ and increased fuel efficiency ${ }^{3,4}$ and, in the future, may provide a means of safely coordinating arrival and departure of commercial aircraft ${ }^{5}$ or increased effectiveness in environmental sampling applications. ${ }^{6,7,8}$ In order to achieve these benefits with autonomous systems, strategies for individual vehicles to sense other aircraft in the formation are needed; using the aerodynamic effects caused by flying in close proximity may be a reliable method. This paper considers a two-aircraft leader-follower formation and employs tools from nonlinear observability, nonlinear estimation, and nonlinear control to enable the follower aircraft to use differential pressure measurements to estimate the leader aircraft's wake and to position itself at a desired location relative to the leader. This work can be applied to instances of close formation flight such as aerial refueling or for increasing aerodynamic efficiency through induced drag reduction. ${ }^{1,3,4}$

An extensive body of literature has been produced regarding the modeling of close formation flight, ${ }^{4}$ from the aerodynamics of birds in formation ${ }^{9}$ to power savings produced by specific aircraft formations. ${ }^{10}$ A large portion of the close-formation modeling, ${ }^{3,11,12}$ control, ${ }^{13,14}$ and experimental ${ }^{15}$ studies have focused on formations that produce significant reductions in the induced drag on an aircraft flying within the wake of another aircraft. Most of these works utilize lifting-line or vortex-lattice methods ${ }^{16,17}$ to model the aerodynamics of aircraft interactions. Closely related work ${ }^{1,2}$ addresses the modeling and experimental study of aerodynamic and dynamical effects related to aerial refueling and presents a feedback controller to stabilize a two-aircraft refueling maneuver.

Success of close-formation flight is predicated on knowledge of the leader aircraft's relative position and the characteristics of its associated wake. One aspect of this paper addresses the problem of estimating characteristics of the leader aircraft wake from its effects on the aerodynamics of the follower aircraft. Similar to Hemati et al., ${ }^{18}$ this paper uses lifting-line theory to model a two-aircraft formation and to estimate parameters of the leader aircraft wake. However, unlike prior work, ${ }^{18}$ this paper implements a grid-based recursive Bayesian filter to estimate the nonlinear wake properties, as opposed to an extended Kalman filter or particle filter; a simple implementation is possible because the follower's kinematics are 
presumed known. In addition, analysis of the nonlinear observability of the leader aircraft wake parameters facilitates the development of two optimal control strategies that steer the follower aircraft to a desired position while accounting for wake observability. These strategies serve to increase estimator performance and, in turn, the overall performance of the control algorithms, which are based on a receding horizon optimal control strategy ${ }^{19}$ and a wavefront propagation strategy. ${ }^{20}$

Tools from nonlinear observability have been used in a wide range of applications based on analytical, numerical, and empirical methods. When applied to close formation flight, observability analysis provides a method of mapping "blind spots" in which the follower aircraft may not be able to estimate the leader aircraft wake parameters because they are highly unobservable. Analytical approaches to nonlinear system observability were introduced by Hermann and Krener ${ }^{21}$ using a Lie algebra to prove local observability over varying time scales. Analytical methods are often complicated for even simple nonlinear systems. Numerical techniques, ${ }^{22,23,24}$ such as the empirical observability gramian employed in this paper, may be easier to implement and often produce comparable results. Empirical methods have been used for model reduction of nonlinear systems ${ }^{22}$ to evaluate candidate sensor placements for observing chemical processes,${ }^{25,26}$ to evaluate the effectiveness of Lagrangian drifter sensors for estimating point vortex flows, ${ }^{23}$ and in adaptive sampling and control for flowfield estimation. $^{27,28}$ To the authors' knowledge this paper is the first instance in which empirical observability measures have been applied to wake sensing in close-formation flight.

The contributions of this paper are (1) a method for quantitative analysis of the nonlinear observability of a leader aircraft's wake parameters given distributed measurements of differential pressure collected by the follower aircraft; (2) a recursive Bayesian filtering framework allowing the follower aircraft to assimilate distributed noisy measurements of differential pressure and a low-fidelity (binary) measurement of relative altitude to resolve the leader aircraft wake parameters; and (3) an optimal control algorithm to steer the follower aircraft to a desired vertical/lateral position relative to the leader aircraft while maximizing observability of the leader's parameters along the trajectory. Control of the along-track separation of the leader and following aircraft is outside the scope of this paper. ${ }^{13}$

The organization of this paper is as follows. Section II uses lifting-line theory to develop a model of two aircraft in formation flight and provides an overview of tools from nonlinear observability, including the empirical observability gramian. Section III presents a recursive Bayesian filter for estimating the leader aircraft wake parameters and formulates two observability-based optimal control strategies to steer the follower aircraft to a desired position relative to the leader. Section IV provides numerical examples of formation flight and aerial refueling maneuvers in which estimates generated by the recursive Bayesian filter are incorporated into the optimal control strategies. Section V provides closing remarks. 


\section{Dynamic Modeling and Leader Aircraft Observability}

This section reviews the aerodynamic modeling of a two-aircraft formation and describes tools from nonlinear observability that are utilized in Section III. In particular, Section II.A provides a two-aircraft aerodynamic model in which the leader's wake affects the aerodynamics of a follower; Section II.B reviews the empirical observability gramian and corresponding measures of observability of the leader's wake parameters. The observability measures are incorporated into the optimal control and estimation results in Section III.

\section{A. Two-Aircraft Aerodynamic Model}

This section describes an aerodynamic model of a follower aircraft flying in a leader aircraft's wake, similar to that of Hemati et al. ${ }^{18}$ and Pachter et al. ${ }^{13}$ Consider two aircraft in steady level flight through an inviscid, incompressible, irrotational fluid. Let the reference frame $\mathcal{B}=\left(O, \boldsymbol{b}_{1}, \boldsymbol{b}_{2}, \boldsymbol{b}_{3}\right)$ with origin $O$ be attached to the center of the leading edge of the follower's wing with basis vectors ${ }^{\mathrm{a}} \boldsymbol{b}_{1}, \boldsymbol{b}_{2}$, and $\boldsymbol{b}_{3}$ as shown in Figure 1 . Assume that the follower aircraft maintains kinematic control of its vertical and horizontal velocities such that the velocity of frame $\mathcal{B}$ with respect to the leader aircraft in steady level flight is $\mathbf{V}_{f}=V_{y} \mathbf{b}_{2}+V_{z} \mathbf{b}_{3}$ (the $\mathbf{b}_{1}$ component is assumed to be zero). The leader has wingspan $b$ and center position $\boldsymbol{r}_{L}=x_{L} \boldsymbol{b}_{1}+y_{L} \boldsymbol{b}_{2}+z_{L} \boldsymbol{b}_{3}$ relative to $O$. Assume $\left|x_{L}\right|$ is sufficiently large (i.e., greater than two wingspans ${ }^{13}$ ) such that the wake of the leader is adequately represented using potential flow theory as the sum of two infinite-line vortices, each with circulation strength $\Gamma_{L}$, extending horizontally behind the wingtips of the leader along the $\boldsymbol{b}_{1}$ direction. The Biot-Savart law gives the following vertical component of the wake ${ }^{3,13,18} w_{L}$ at a point $(x, y, z)$ along the $\boldsymbol{b}_{2}$ axis $(x=z=0)$ as a function of the leader's position ${ }^{\mathrm{b}}$ :

$$
w_{L}\left(y ; \Gamma_{L}, y_{L}, z_{L}, b\right)=\frac{\Gamma_{L}\left(y-y_{L}-b / 2\right)}{2 \pi\left(z_{L}^{2}+\left(y-y_{L}-b / 2\right)^{2}\right)}-\frac{\Gamma_{L}\left(y-y_{L}+b / 2\right)}{2 \pi\left(z_{L}^{2}+\left(y-y_{L}+b / 2\right)^{2}\right)}
$$

Note that (1) is symmetric about $z_{L}=0$. This property necessitates invoking a second sensor later in the paper to break the vertical symmetry. The aerodynamic signature on the follower aircraft created by the upwash field of the leader will be used to estimate the two-dimensional position $\mathbf{r}=\left(y_{L}, z_{L}\right)$ of the leader relative to the follower and the circulation strength $\Gamma_{L}$ of the leader's wake. The position estimate $\hat{\mathbf{r}}$ will be used in an optimal controller to steer the follower to the desired relative position.

The flow around the follower aircraft in response to the upwash field of the leader is

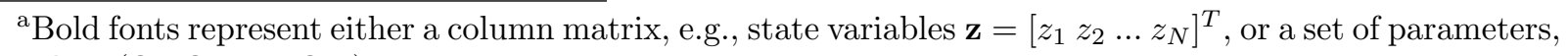
e.g., $\boldsymbol{\Omega}=\left(\Omega_{1}, \Omega_{2}, \ldots, \Omega_{M}\right)$.

${ }^{\mathrm{b}}$ The notation $g(a, b ; \alpha, \beta)$ represents a function $g(\cdot)$ that depends on the state variables $a, b$ and the parameters $\alpha$ and $\beta$.
} 


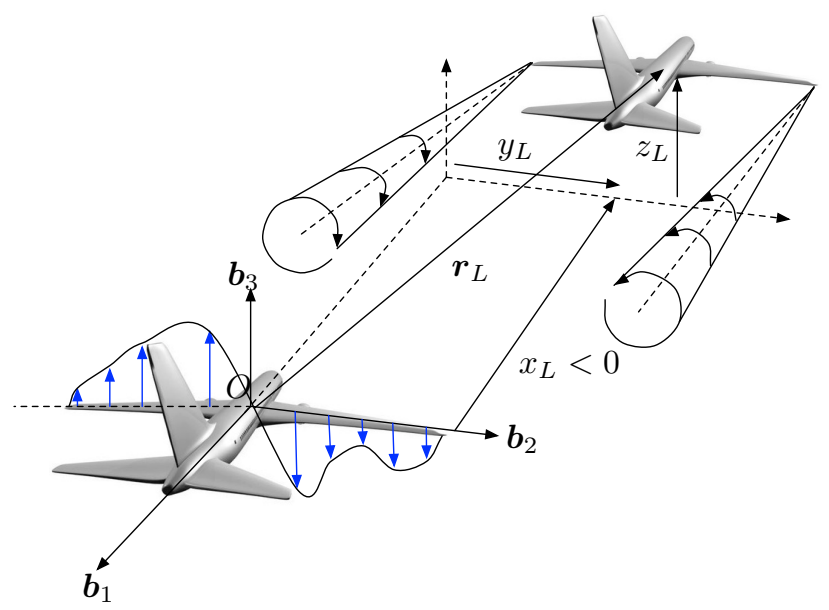

Figure 1. Leader and follower aircraft representations. The wake of the leader produces an aerodynamic signature on the follower through its upwash field (blue).

modeled by employing the lifting-line solution following Katz and Plotkin [16, p. 331-340]. For simplicity, assume the follower is represented by a flat, thin, rectangular wing with large aspect ratio $(R>4)$ and chord length $c$ as shown in Figure 2. (Note this method is capable of modeling more complex wing geometries, including swept and dihedral wings. ${ }^{16}$ ) Since the fluid is inviscid, incompressible, and irrotational, its motion can be represented by the gradient of a potential function $\Phi$ that satisfies Laplace's equation ${ }^{16}$

$$
\nabla^{2} \Phi=0
$$

In addition, at every point on the wing the potential function $\Phi$ must satisfy the boundaryvalue constraint, which ensures that there is no flow normal to the wing surface. Assuming the wing is thin, its normal vector $\mathbf{n}_{i}$ at any point $\left(x_{i}, y_{i}, z_{i}\right)$ on the wing surface is approximately $\mathbf{n}_{i} \approx \mathbf{b}_{3}, i=1, \ldots, N$, which implies

$$
\nabla \Phi \cdot \mathbf{b}_{3}=0
$$

as shown in Figure 2. To satisfy these constraints, lifting-line theory constructs a suitable potential function from a collection of line vortices. $N$ equally spaced horseshoe vortices are bound to the quarter chord of the follower aircraft wing such that $2 N$ trailing vortices extending infinitely downstream. The $i^{\text {th }}$ bound horseshoe vortex has circulation strength $\Gamma_{i}$ as shown in Figure 2. The number $N$ of horseshoe vortices must be chosen large enough for adequate model fidelity, yet small enough to remain computationally tractable. The freestream fluid velocity $\mathbf{U}_{\infty}$ has magnitude $U_{\infty}$ and angle of attack $\alpha$ relative to the wing. (Assume the freestream velocity has zero sideslip and $\alpha$ is small.)

The line vortex is a solution to Laplace's equation ${ }^{16}(2)$, implying that the flow due to the 


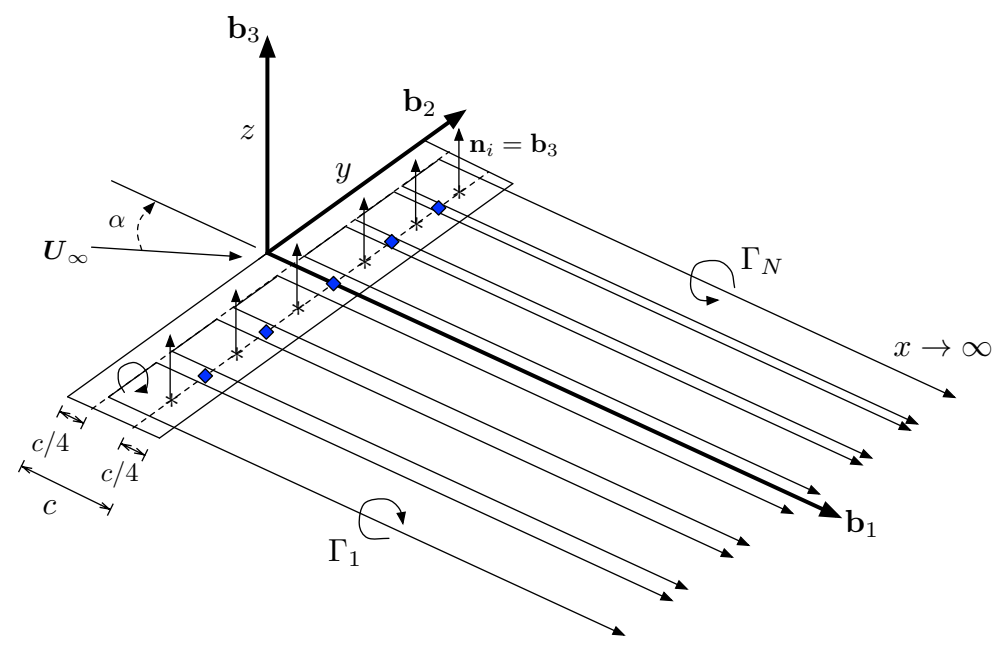

Figure 2. Horseshoe vortices are used to model the flow around a finite, slender wing with aspect ratio $\boldsymbol{R}>4$ representing the follower aircraft.

freestream velocity, leader aircraft upwash, and horseshoe vortices must satisfy the normal flow constraint (3). Therefore, (3) evaluated at any given point on the wing must satisfy

$$
w_{h s}+w_{L}+V_{z}+U_{\infty} \sin \alpha=0
$$

where $w_{h s}$ is the $\mathbf{b}_{3}$ component of the flow generated by the horseshoe vortices, $w_{L}$ is the vertical component of the leader aircraft's wake given by (1), $V_{z}$ is the $\mathbf{b}_{3}$ component of the inertial velocity of the follower aircraft expressed in frame $\mathcal{B}$, and the fourth term on the lefthand side is the normal component of the freestream velocity. Note that this model neglects aerodynamic influences due to aircraft pitching, rolling, and sideslip maneuvers, under the assumption that in close proximity these motions are negligible relative to the aerodynamic effect of vertical motion.

To solve for the horseshoe vortex strengths $\Gamma_{i}$ that satisfy (4), this paper employs the collocation method. ${ }^{16,17}$ Following Katz and Plotkin [16, p. 331-334], impose the constraint (4) at $N$ collocation points centered at each horseshoe vortex along the $3 / 4$-chord line as shown in Figure 2. Since the flow at any collocation point is linearly dependent on the circulation strength $\Gamma_{i}$ of the $i^{\text {th }}$ horseshoe vortex, (4) applied at the $N$ collocation points forms a set of $N$ linear algebraic equations with $N$ unknown circulation strengths ${ }^{16} \Gamma_{i}$, $i=1 \ldots, N$,

$$
\left[\begin{array}{ccc}
a_{11} & \cdots & a_{1 N} \\
\vdots & \ddots & \vdots \\
a_{N 1} & \cdots & a_{N N}
\end{array}\right]\left[\begin{array}{c}
\Gamma_{1} \\
\vdots \\
\Gamma_{N}
\end{array}\right]=-\left(U_{\infty} \sin \alpha+V_{z}\right)\left[\begin{array}{c}
1 \\
\vdots \\
1
\end{array}\right]-\left[\begin{array}{c}
w_{L}\left(y_{1}\right) \\
\vdots \\
w_{L}\left(y_{N}\right)
\end{array}\right]
$$


Here

$$
\sum_{i=1}^{N} a_{j i} \Gamma_{i}=w_{h s}\left(y_{j}\right),
$$

is the normal component of the flow at the $j^{\text {th }}$ collocation point, $V_{z}$ is the (control) velocity of the follower aircraft, and $w_{L}\left(y_{j}\right)$ is obtained by evaluating (1) at $y_{j}$. Equation (5) is solved for the circulation strength distribution $\Gamma_{1}, \ldots, \Gamma_{N}$ by inverting the $a_{j i}$ coefficient matrix. ${ }^{16}$

The circulation strength distribution $\Gamma(y)=\lim _{N \rightarrow \infty} \Gamma_{i}$ is used to calculate measurable quantities that will be used in the nonlinear wake estimation process. For example, Hemati et al. ${ }^{18}$ assume the following measurements of the differential pressure coefficient $\Delta C_{p}$ :

$$
\Delta C_{p}(x, y)=\frac{-4 \Gamma(y)}{\pi U_{\infty} c}\left(\frac{c}{x}-1\right)^{1 / 2}
$$

Assume equally spaced measurements of differential pressure on the $3 / 4$-chord line $x=(3 c) / 4$ along the span of the wing, as shown by the blue squares in Figure 2.

Let $\mathbf{h}(\Omega)=\left[\Delta C_{p 1} \ldots \Delta C_{p P}\right]^{T}$ be a $P \times 1$ column matrix of differential pressure measurements calculated using (7) on the 3/4-chord line as shown in Figure 2, where $P$ is the number of measurements. Taking $\boldsymbol{h}(\boldsymbol{\Omega})$ as the output and using the leader aircraft dynamics in frame $\mathcal{B}$, generates the following state-space form of the input-output relationship between the wake parameter states $\Omega=\left[y_{L}, z_{L}, \Gamma_{L}\right]^{T}$ and the measurements $\boldsymbol{\phi}$ :

$$
\begin{aligned}
& \dot{\boldsymbol{\Omega}}=\left[\begin{array}{c}
-V_{y} \\
-V_{z} \\
0
\end{array}\right] \\
& \boldsymbol{\phi}=\mathbf{h}(\boldsymbol{\Omega}) .
\end{aligned}
$$

The model (8) will be used to evaluate the observability of the state $\Omega$ with the output equation $\mathbf{h}(\boldsymbol{\Omega})$, to design an observer to estimate $\boldsymbol{\Omega}$ from noisy output measurements, and to design optimal control strategies for the follower aircraft.

\section{B. Measures of Leader Aircraft Observability}

Successful estimation of states in a system depends on the ability to observe the desired states from the sensor measurements collected over time. ${ }^{23}$ This paper seeks to observe the wake parameter states $\Omega=\left[y_{L}, z_{L}, \Gamma_{L}\right]^{T}$ given measurements of the differential pressure coefficient in (7). Equation (7) depends on $\Omega$ indirectly through the circulation strength distribution $\Gamma(y)$ of the horseshoe vortices, which motivates the need to quantitatively assess the observability of the parameters $\Omega$ as part of the implementation of an observer-based controller. This section provides a brief overview of observability in the context of linear and 
nonlinear systems and reviews the empirical observability gramian ${ }^{22,23,27}$ used to assess the observability of the wake parameters in the aerodynamic model of Section II.A.

A dynamical system is said to be observable if its initial conditions can be determined from a time history of output measurements $\mathbf{h}(t)$ and control inputs $\mathbf{u}(t)$ over some time interval. $^{21,29}$ A standard method of measuring observability is to calculate the singular values of the observability gramian. ${ }^{26,29}$ In linear systems theory, the singular values of the observability gramian quantify the ease in determining the initial states from the outputs generated over time as follows: large singular values imply the mapping is easily invertible, whereas small or zero singular values imply it is not [29, p. 125-126]. This paper quantifies the unobservability of a system using the unobservability index $\xi$, which is the reciprocal of the smallest singular value $\sigma_{m i n}$, i.e., ${ }^{23,27}$

$$
\xi=\frac{1}{\sigma_{\min }} .
$$

The unobservability index (9) reflects the least observable mode in the system and provides a worst-case observability measure for the system. Another measure is the estimation condition number $\lambda$, defined as the ratio of the largest and smallest singular values ${ }^{23,26,30}$

$$
\lambda=\frac{\sigma_{\max }}{\sigma_{\min }}
$$

which characterizes the range of observability in the system. A large value of $\lambda$ implies the estimation problem may be ill-conditioned, ${ }^{23,27,30}$ the minimum value of $\lambda$ is one.

The observability of a nonlinear system may be difficult to determine analytically, because it requires tools from differential geometry. ${ }^{21}$ Since the flow model in this paper is solved numerically, it is justified to pursue numerical techniques for calculating the nonlinear, empirical observability gramian. ${ }^{24,30}$ Linearization may fail to adequately model the input/output relationship of the nonlinear system over a wide range of operating conditions. The empirical observability gramian does not require linearization but merely the ability to simulate the system. Indeed, the empirical observability gramian maps the input-output behavior of a nonlinear system more accurately than the observability gramian produced by linearization of the nonlinear system. ${ }^{31}$

The empirical observability gramian is defined as follows. Let $\epsilon_{i} \mathbf{e}_{i}, i=1, \ldots, M$, be a small displacement of the nominal parameter along the $i^{\text {th }}$ unit vector $\mathbf{e}_{i} \in \mathbb{R}^{M}$ and let $\Omega \in \mathbb{R}^{M}$ be the set of nominal parameter values. The $(i, j)^{\text {th }}$ component of the $M \times M$ empirical observability gramian $W_{O}$ is ${ }^{23}$

$$
\begin{aligned}
W_{O}(i, j)= & \frac{1}{4 \epsilon_{i} \epsilon_{j}} \int_{0}^{T}\left[\boldsymbol{\phi}^{+i}(\tau)-\boldsymbol{\phi}^{-i}(\tau)\right]^{T}\left[\boldsymbol{\phi}^{+j}(\tau)-\boldsymbol{\phi}^{-j}(\tau)\right] \mathrm{d} \tau \\
& i=1, \ldots, M, j=1, \ldots, M
\end{aligned}
$$


where $\Omega^{ \pm i}=\Omega \pm \epsilon_{i} \mathbf{e}_{i}$ produces the output $\phi^{ \pm i}=\mathbf{h}\left(\Omega^{ \pm i}, \mathbf{u}\right)$. Measures of the observability of a nonlinear system are obtained by applying (9) and (10) to $W_{O}$.

The empirical observability gramian is used here to evaluate the observability of the leader aircraft wake parameters along trajectories of relative aircraft motion. This analysis provides a method of mapping "blind spots" in which the follower aircraft may not be able to estimate the leader's wake parameters because they are highly unobservable. To analyze the dependence of the unobservability index and estimation condition on the leader aircraft position, the empirical observability gramian (11) is evaluated as a function of the leader aircraft position in the $\left(\mathbf{b}_{2}, \mathbf{b}_{3}\right)$ plane.

For simulation purposes, assume $N=40$ horseshoe vortices define the aircraft wing model. The wing dimensions and flight conditions are based on a C-17 aircraft $(b=51.75$ meters, $R=7.586$, cruise $U_{\infty}=230.556 \mathrm{~m} / \mathrm{s}$ ). All quantities are non-dimensionalized using wingspan and cruise speed, so they can be generalized to other (unmanned) aircraft. Figure 3 shows the $\log$ of the observability measures (9) and (10) at each relative position $\mathbf{r}=\left[\begin{array}{lll}y_{L} & z_{L}\end{array}\right]^{T}$. Note that the unobservability index in Figure 3(a) generally increases with increasing $\|\mathbf{r}\|$ and is symmetric in the $\left(\mathbf{b}_{2}, \mathbf{b}_{3}\right)$ plane, which is expected due to the symmetries of the upwash model (1). However, pockets of high unobservability (poor observability) extend outward from the wing tips of the follower. As a result, there is a large degree of variability in observability between one and two wingspans from the origin of the $\left(\mathbf{b}_{2}, \mathbf{b}_{3}\right)$ plane. Further, the unobservability index is nearly seven orders of magnitude larger two wingspans away than at the origin, implying that one can expect dramatically worse estimation performance as $\|\mathbf{r}\|$ increases; this pattern is not radially symmetric. Additional calculations (not shown) indicate that the singular value associated with the circulation strength parameter $\Gamma_{L}$ is typically the smallest, which implies that $\Gamma_{L}$ is the least observable state in $\Omega$ and the most difficult to estimate. Since the estimation condition in Figure 3(b) is large along diagonals emanating outward from the wingtips, the estimation problem is poorly conditioned in these areas.

Evaluation of the empirical observablity gramian over the space of leader aircraft positions provides an observability map of the leader aircraft parameters $\Omega$. The observability analysis is used in the following section to guide the design of a recursive Bayesian filter for estimating the leader's wake parameters and two observability-based optimal control strategies to steer the follower to a desired relative position. Maintaining adequate observability guarantees the performance of the filtering scheme. 

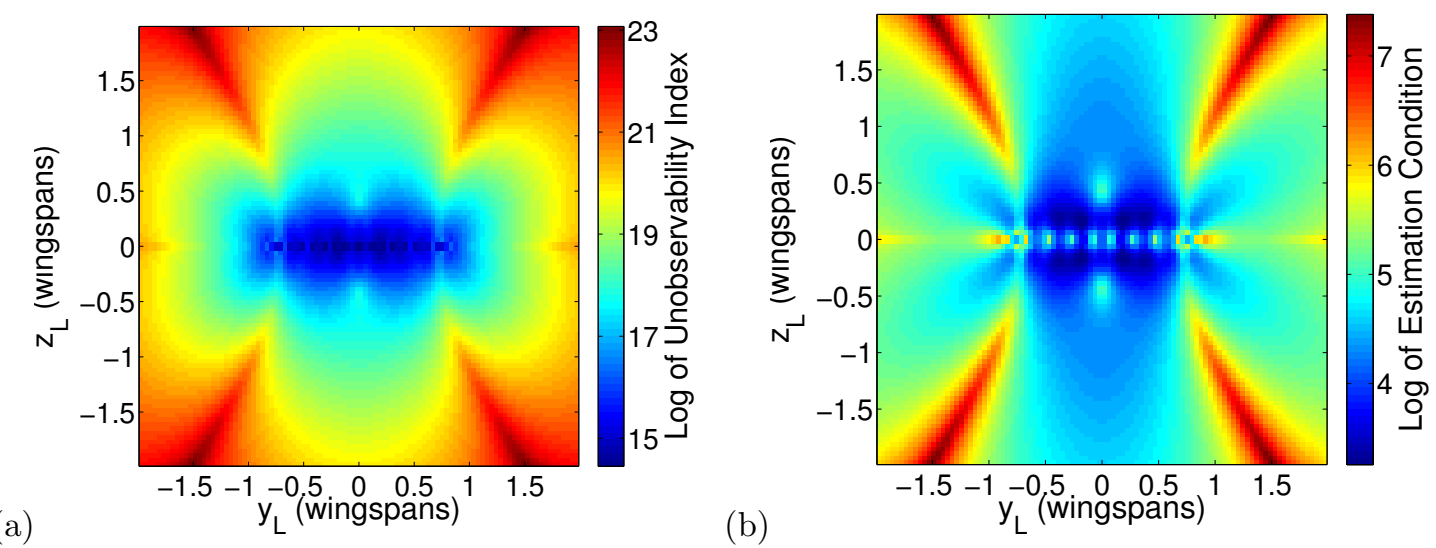

Figure 3. The log of the (a) unobservability index and (b) estimation condition over a range of leader aircraft relative positions.

\section{Wake Estimation and Control}

This section presents a recursive Bayesian filter for estimating the leader aircraft states of the two-aircraft model (8) and derives two optimal control algorithms to steer the follower to a desired position relative to the leader. The leader's estimated states are utilized in the optimal control. Numerical examples are provided in Section IV.

\section{A. Bayesian Estimation of Leader Aircraft Wake Parameters}

For a linear system with Gaussian noise, the optimal Bayesian filter is the Kalman filter, whereas for a nonlinear system with nonlinear noise, an effective Bayesian filter is a particle filter. ${ }^{32}$ Hemati et. al ${ }^{18}$ compared the performance of an extended Kalman filter with a particle filter in estimating the leader aircraft wake parameters. Due to the fact that there are only a few states and each have a known time dependence, a grid-based recursive Bayesian estimation scheme is selected here to assimilate the differential pressure coefficient measurements with noise. The state vector $\Omega$ contains a set of $M=3$ states $\Omega=\left[\begin{array}{lll}y_{L} & z_{L} \Gamma_{L}\end{array}\right]^{T}$, from which the differential pressure distribution is reconstructed. The leader aircraft wake model (1) has kinematics that are modeled in (nonlinear) state-space form in (8).

The grid-based, discrete-time Bayesian formalism is presented as follows. ${ }^{32}$ Let $\boldsymbol{\Omega}(t)$ denote the state estimate at time $t, \tilde{\boldsymbol{\mu}}(t)$ denote noisy sensor observations at time $t$, and $\mathbf{A}(t)=\{\tilde{\boldsymbol{\mu}}(1), \ldots, \tilde{\boldsymbol{\mu}}(t)\}$ denote the set of observations up to time $t$. The posterior probability of the state $\boldsymbol{\Omega}(t)$ given $\mathbf{A}(t)$ evolves in time via ${ }^{33}$

$$
p(\boldsymbol{\Omega}(t) \mid \mathbf{A}(t))=\beta p(\tilde{\boldsymbol{\mu}}(t) \mid \boldsymbol{\Omega}(t)) \int p(\boldsymbol{\Omega}(t) \mid \boldsymbol{\Omega}(t-\Delta t)) p(\boldsymbol{\Omega}(t) \mid \mathbf{A}(t-\Delta t)) \mathrm{d} \boldsymbol{\Omega}(t-\Delta t),
$$

where the coefficient $\beta$ is chosen so that $p(\boldsymbol{\Omega}(t) \mid \mathbf{A}(t))$ has unit integral over the state space. 
The conditional probability $p(\tilde{\boldsymbol{\mu}}(t) \mid \boldsymbol{\Omega}(t))$ is a likelihood function that represents the probability of the state $\boldsymbol{\Omega}(t)$ given the observation $\tilde{\boldsymbol{\mu}}(t)$. The motion model $p(\boldsymbol{\Omega}(t) \mid \boldsymbol{\Omega}(t-\Delta t))$ represents a nonlinear operator that updates the probability density function from $t-\Delta t$ to $t$ [33, p. 372-375], assuming known control inputs $\mathbf{u}=\left[V_{y} V_{z}\right]^{T}$. Define the motion matrix $\Psi=\Delta t \operatorname{diag}\left(\left[-V_{y}-V_{z} 0\right]^{T}\right)$ and let $p(\Omega(t) \mid \Omega(t-\Delta t))=\mathcal{N}\left(\Psi \Omega(t-\Delta t) ; \Sigma_{p}\right)$, where $\mathcal{N}\left(\Psi \Omega(t-\Delta t) ; \Sigma_{p}\right)$ is normally distributed white noise with mean $\Psi \Omega(t-\Delta t)$ and variance $\Sigma_{p}$. The quantity $p(\boldsymbol{\Omega}(t) \mid \mathbf{A}(t-\Delta t))$ is the prior probability density resulting from measurements taken up to $t-\Delta t$. At $t=0$ the probability is assumed to be uniformly distributed in the absence of information other than the parameter lower and upper bounds. The maximum likelihood estimate $\hat{\Omega}$ of the leader aircraft parameters is the point in parameter space corresponding to the maximum of the posterior probability density, i.e.,

$$
\hat{\mathbf{\Omega}}=\sup p(\boldsymbol{\Omega}(t) \mid \mathbf{A}(t))
$$

Suppose the sensors collect noisy measurements of differential pressure according to

$$
\tilde{\boldsymbol{\phi}}(t)=\boldsymbol{\phi}(t)+\boldsymbol{\eta}_{\phi}(t) \in \mathbb{R}^{P}
$$

where the noise $\boldsymbol{\eta}_{\phi}(t) \sim \mathcal{N}\left(0, \Sigma_{\phi}^{2}\right)$ is a $P \times 1$ column matrix in which each element has zero mean and variance $\Sigma_{\phi}^{2}$, and $\phi$ is given by (8). To break the vertical symmetry in (1), assume the follower aircraft is outfitted with a sensor that provides a (noisy) reading of the sign of $z_{L}$, i.e.,

$$
\begin{gathered}
\theta(t)=\operatorname{sgn}\left(z_{L}(t)\right)=\left\{\begin{array}{cc}
1, & z_{L}(t)>0 \\
0, & z_{L}(t)=0 \\
-1 & z_{L}(t)<0
\end{array}\right. \\
\tilde{\theta}(t)=\theta(t)+\eta_{\theta}(t)
\end{gathered}
$$

where the noise $\eta_{\theta}$ has zero mean and variance $\Sigma_{\theta}^{2}$. Multi-variate Gaussian likelihood functions are chosen for each point $\Omega(t)$ in the $M$-dimensional state space to fuse contributions from both sensing types, i.e.,

$$
p(\boldsymbol{\mu}(t) \mid \boldsymbol{\Omega}(t))=p(\boldsymbol{\phi}(t) \mid \boldsymbol{\Omega}(t)) p(\theta(t) \mid \boldsymbol{\Omega}(t))
$$

where $\boldsymbol{\mu}=(\boldsymbol{\phi}, \theta)$ combines both sensing modalities. The likelihood functions for the differential pressure and relative altitude measurements are

$$
p(\boldsymbol{\phi}(t) \mid \boldsymbol{\Omega}(t))=\frac{1}{2 \pi \Sigma_{\phi}^{1 / 2}} \exp \left[-\frac{1}{2 \Sigma_{\phi}^{2}}[\mathbf{h}(\boldsymbol{\Omega}(t))-\boldsymbol{\phi}(t)]^{T}[\mathbf{h}(\boldsymbol{\Omega}(t))-\boldsymbol{\phi}(t)]\right]
$$


and

$$
p(\theta(t) \mid \boldsymbol{\Omega}(t))=\frac{1}{2 \pi \Sigma_{\theta}^{1 / 2}} \exp \left[-\frac{1}{2 \Sigma_{\theta}^{2}}\left(\operatorname{sgn}\left(z_{L}(\boldsymbol{\Omega}(t))\right)-\theta(t)\right)^{2}\right],
$$

respectively. The term $z_{L}(\boldsymbol{\Omega}(t))$ in (17) represents the $z_{L}$ value associated with the state $\boldsymbol{\Omega}(t)$ and $\Sigma_{\theta}^{2}$ is the variance in the relative altitude measurement. Estimates from the recursive Bayesian filter are used in the optimal controllers presented in the following section.

\section{B. Observability-based Optimal Control}

Section II.B showed how the unobservability index $\xi=\xi(\mathbf{r})$ and estimation condition $\lambda=$ $\lambda(\mathbf{r})$ quantify the observability over the relative position space $\mathbf{r}$. The observability map can be viewed as a cost metric for use in designing an optimal control strategy that steers the follower aircraft to a desired relative position while maintaining adequate observability along the trajectory. Maintaining adequate observability along a trajectory guarantees the performance of the estimation scheme, which increases the control algorithm performance. This section presents the control derivation as an optimal control problem and provides two methods of control design. The first method combines observability and the control-signal magnitude to form a cost metric and solves a receding-horizon optimal control problem with numerical methods. The second strategy employs a wavefront expansion method ${ }^{20}$ to quickly generate solutions over the discretized space of leader aircraft positions and incorporates gradient descent control to produce optimal follower aircraft trajectories.

The classical optimal control problem is formulated as follows. Suppose that for aerodynamic efficiency or aerial refueling purposes the follower aircraft must maintain a desired position relative to the leader aircraft $\mathbf{r}_{d e s}=\left[y_{d e s}, z_{d e s}\right]^{T}$, assuming the longitudinal separation between the aircraft $x_{L}$ is held fixed through a separate control strategy not described here. The control kinematics of the leader aircraft position with respect to the follower are given by (8). Without loss of generality, neglecting the time-invariant quantity $\Gamma_{L}$ in $(8)$ and considering only the relative position terms $\mathbf{r}$, yields the equations of motion

$$
\dot{\mathbf{r}}=\mathbf{f}(\mathbf{u})=\left[\begin{array}{c}
-V_{y} \\
-V_{z}
\end{array}\right]
$$

where $\mathbf{r}=\left[\begin{array}{ll}y_{L} & z_{L}\end{array}\right]^{T}$ and $\mathbf{u}=\left[\begin{array}{ll}V_{y} & V_{z}\end{array}\right]^{T}$.

The desired control $\mathbf{u}(t)$ produces a trajectory optimizing observability from the initial condition $\mathbf{r}\left(t_{0}\right)=\left[y_{L}\left(t_{0}\right), z_{L}\left(t_{0}\right)\right]^{T}$ at time $t_{0}$ to the desired position $\mathbf{r}_{\text {des }}$ at or before time $t_{0}+T_{f}$. Consider the receding-horizon cost function

$$
J_{R H}\left(\mathbf{r}\left(t_{0}\right) ; \kappa, \mathbf{R}\right)=\int_{t_{0}}^{t_{0}+T_{f}}\left(\kappa \log \xi(\mathbf{r}(t))+\mathbf{u}(t)^{T} \mathbf{R u}(t)\right) \mathrm{d} t
$$



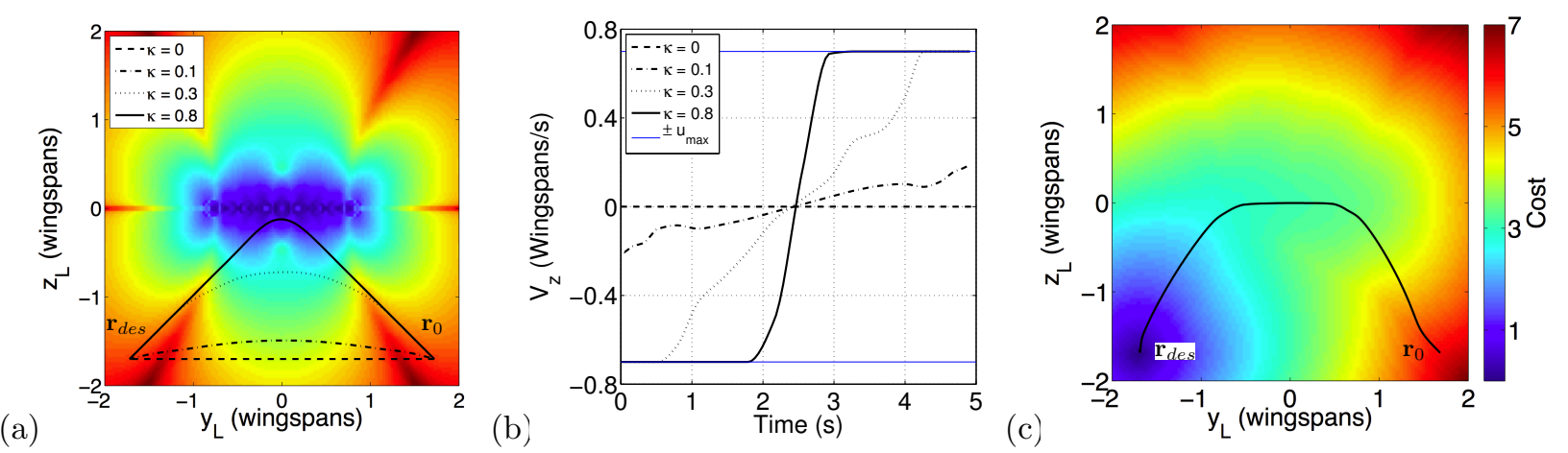

Figure 4. (a) Optimal trajectory solutions of the cost function (19) and (b) corresponding $V_{z}$ component of the control $\mathbf{u}(t)$ for initial condition $\mathbf{r}_{0}=\left[\begin{array}{ll}1.7 & -1.7\end{array}\right]^{T}$ wingspans and desired

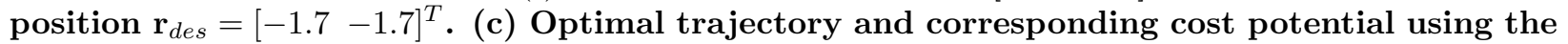
fast marching method.

where $\xi(\mathbf{r})$ is the unobservability index (9). The constant weights $\mathbf{R} \in \mathbb{R}^{2 \times 2}$ and $\kappa \in \mathbb{R}$ are positive definite. The cost function (19) is positive semi-definite since $\xi(\mathbf{r}) \geq 0$ by definition and, in this application, its natural $\log$ is also positive definite since $\sigma_{m i n} \leq 1$ for the parameter values used. Minimizing (19) is a constrained nonlinear optimization problem whose solution provides the control sequence $\mathbf{u}(t)$ that produces the optimal observability trajectory with minimum control effort. Imposing the constraint $\|\mathbf{u}(t)\| \leq u_{\max }=\sqrt{V_{y_{\max }}^{2}+V_{z_{\max }}^{2}}$ bounds the control effort. Using the GPOPS ${ }^{19,34}$ optimization software in MATLAB yields the controls $V_{y}^{*}$ and $V_{z}^{*}$ that minimize $J_{R H}$.

Figure 4(a) depicts the optimal trajectories of the leader aircraft for $\kappa=0,0.1,0.3$, and 0.8 , and $\mathbf{R}=I_{2 \times 2}$. The initial condition $\mathbf{r}_{0}=(1.7,-1.7)$ wingspans and desired position $\mathbf{r}_{\text {des }}=(-1.7,-1.7)$ wingspans are arbitrary. As $\kappa$ increases, the observability along the trajectory improves. Figure $4(\mathrm{~b})$ shows the $V_{z}$ component of the control signal $\mathbf{u}(t)$ versus time. Decreasing $\kappa$ causes the trajectory to approach the desired location more directly, but via a route that increases unobservability. The $V_{y}$ component maintains its maximum value $V_{y_{\max }}=0.7$ wingspans $/ \mathrm{s}$ for all solutions.

The control $\mathbf{u}(t)$ that minimizes (19) produces the optimal observability trajectory for a given horizon time $T_{f}$ and initial condition $\mathbf{r}_{0}$. To incorporate state estimates from the recursive Bayesian filter, one might use the estimated leader aircraft states $\hat{\mathbf{r}}_{0}$ as the initial condition in the control calculation and recalculate the control iteratively in the following sense. At the start of each planning cycle of duration $T_{e}$ the optimal trajectory to $\mathbf{r}_{d e s}$ is computed using the estimated initial condition $\hat{\mathbf{r}}_{0}$. The follower executes the optimal control $V_{y}^{*}(t), V_{z}^{*}(t)$ for $t \in\left[t_{0}, t_{0}+T_{e}\right]$, then the cycle repeats. Incorporating observability into the optimal control calculation ensures the performance of the recursive filter. As the estimates converge, the follower aircraft approaches the optimal trajectory.

The receding-horizon optimal control strategy presented above requires iterative calcula- 
tion of the optimal control, which is computationally expensive. It also requires specification of horizon times that may be too large or infeasible depending on the leader aircraft's relative position. An alternative approach incorporates a weighted wavefront expansion, known as the fast marching method, ${ }^{20}$ to generate an optimal "cost-to-go" potential map relative to the desired position. The gradient of this potential function provides the optimal control with respect to a given cost function. This method does not require iterative calculation of the control since the potential is calculated over the entire relative position space, making the control computationally inexpensive compared to the receding-horizon control.

The optimal path planning problem is formulated as follows [20, pg. 284-291]. Given a desired position $\mathbf{r}_{\text {des }}$, the goal is to find the path $\mathcal{L}(l):[0, \infty) \rightarrow \mathbb{R}^{2}$ from $\mathbf{r}_{\text {des }}$ to any point $\mathbf{r}_{0}$ that minimizes the observability-based cost integral ${ }^{20}$

$$
\int_{\mathbf{r}_{\text {des }}}^{\mathbf{r}_{0}} \log \xi(\mathcal{L}(l)) \mathrm{d} l,
$$

where $l$ is the arc-length parameterization of the path $\mathcal{L}$ and $\xi(\cdot)$ is the unobservability index evaluated along $\mathcal{L}$. Let the minimum cost required to travel from $\mathbf{r}_{\text {des }}$ to a point $\mathbf{r}$ be $\mathrm{e}^{20}$

$$
J_{W F}(\mathbf{r}) \triangleq \min _{\mathcal{L}} \int_{\mathbf{r}_{\text {des }}}^{\mathbf{r}} \xi(\mathcal{L}(l)) \mathrm{d} l,
$$

such that the level set $J_{W F}(\mathbf{r})=C$ is the set of points that can be reached with minimal cost $C$. By construction, level sets are orthogonal to the minimal cost paths ${ }^{20}$ implying that the optimal path descends the gradient of $J_{W F}(\mathbf{r})$.

The fast marching method ${ }^{20}$ is a wavefront propagation technique that is used to efficiently compute $J_{W F}(\mathbf{r})$ for the domain around the leader aircraft [20, pg. 86-99]. Figure $4(\mathrm{c})$ shows the cost potential $(20)$ with $\mathbf{r}_{\text {des }}=(-1.7,-1.7)$ wingspans. Note that each contour of the potential function indicates the locus of points that can reach $\mathbf{r}_{\text {des }}$ with equal unobservability along the optimal path. The optimal path from $\mathbf{r}_{0}=(1.7,-1.7)$ wingspans is denoted by the black line and follows the gradient of the potential function. This path planning approach does not utilize the magnitude of the control in its cost function and therefore differs from the paths generated previously.

Since the optimal path descends the gradient of $J_{W F}(\mathbf{r})$, an observer-based feedback control incorporates estimates of the relative position $\hat{\mathbf{r}}$ according to

$$
\mathbf{u}=-K_{W F} \nabla J_{W F}(\hat{\mathbf{r}})
$$

where the gain $K_{W F}>0$. Assuming the desired final location is fixed, the cost potential $J_{W F}(\mathbf{r})$ need be calculated only once to produce all possible optimal paths. 


\section{Wake Estimation and Control Examples}

This section provides numerical simulations of the observability-based receding-horizon control and wavefront-propagation control strategies presented in Section III. The control strategies are applied to two examples of close formation flight: The first considers a twoaircraft formation in which the follower aircraft steers itself to a position that will increase aerodynamic efficiency ${ }^{13}$ the second example simulates the follower aircraft positioning itself for aerial refueling. Both simulations assume noise $\Sigma=10^{-5}$ in the differential pressure coefficient measurements and total simulation time $T=15$ seconds. The receding-horizon controller optimization constant is $\kappa=2$, and the horizon time is $T_{f}=5$ seconds for both scenarios. The control optimization calculation is iterated every second, i.e., $T_{e}=1$ second, assuming the recursive Bayesian filter assimilates measurements at $5 \mathrm{~Hz}$. The wavefrontpropagation control strategy has gain $K_{W F}=5$ for all simulations.

\section{A. Two-aircraft Formation Flight}

This section implements the estimation and control algorithms of Section III to steer the two-aircraft model (8) into a formation that increases aerodynamic efficiency for the follower

aircraft. The desired final orientation $\mathbf{r}_{\text {des }}=\left[\frac{\pi b}{4} 0\right]^{T}$ wingspans reduces the induced drag on the follower aircraft, increasing its fuel efficiency. ${ }^{13}$ The initial condition $\mathbf{r}_{0}=(-0.8,1.4)$ wingspans is chosen arbitrarily. Figures 5(a)-(c) illustrate the result of simulating the receding-horizon control algorithm. Figure 5(a) shows the leader aircraft trajectory (white) and the unobservability index. The white circle represents the final position of the leader aircraft and the magenta circle represents the final position estimate. Figures 5(b,c) show the marginal probability density ${ }^{\mathrm{c}}$ of the estimated relative position over time. (The marginal probability density of $\Gamma_{L}$ is omitted for brevity.) A solid white line represents the trajectory of the leader aircraft and a dashed white line shows the trajectory of the state estimate. Note in Figure 5(a) that the leader aircraft is generally steered toward $\mathbf{r}_{\text {des }}$ with deviations from a direct path created by estimation errors in the observability-based control optimization. Figures $5(\mathrm{~b}, \mathrm{c})$ show the marginal probability densities converging to the true aircraft states. Figures 5(d)-(f) illustrate the results of simulating the wavefront-propagation control algorithm. Comparing Figures 5(a) and 5(d), the wavefront-propagation algorithm steers the vehicle along a route to the desired position with fewer deviations due to estimation error than the receding-horizon algorithm.

${ }^{\mathrm{c}}$ The marginal probability density is achieved by summing a multi-dimensional probability density over a subset of dimensions. 

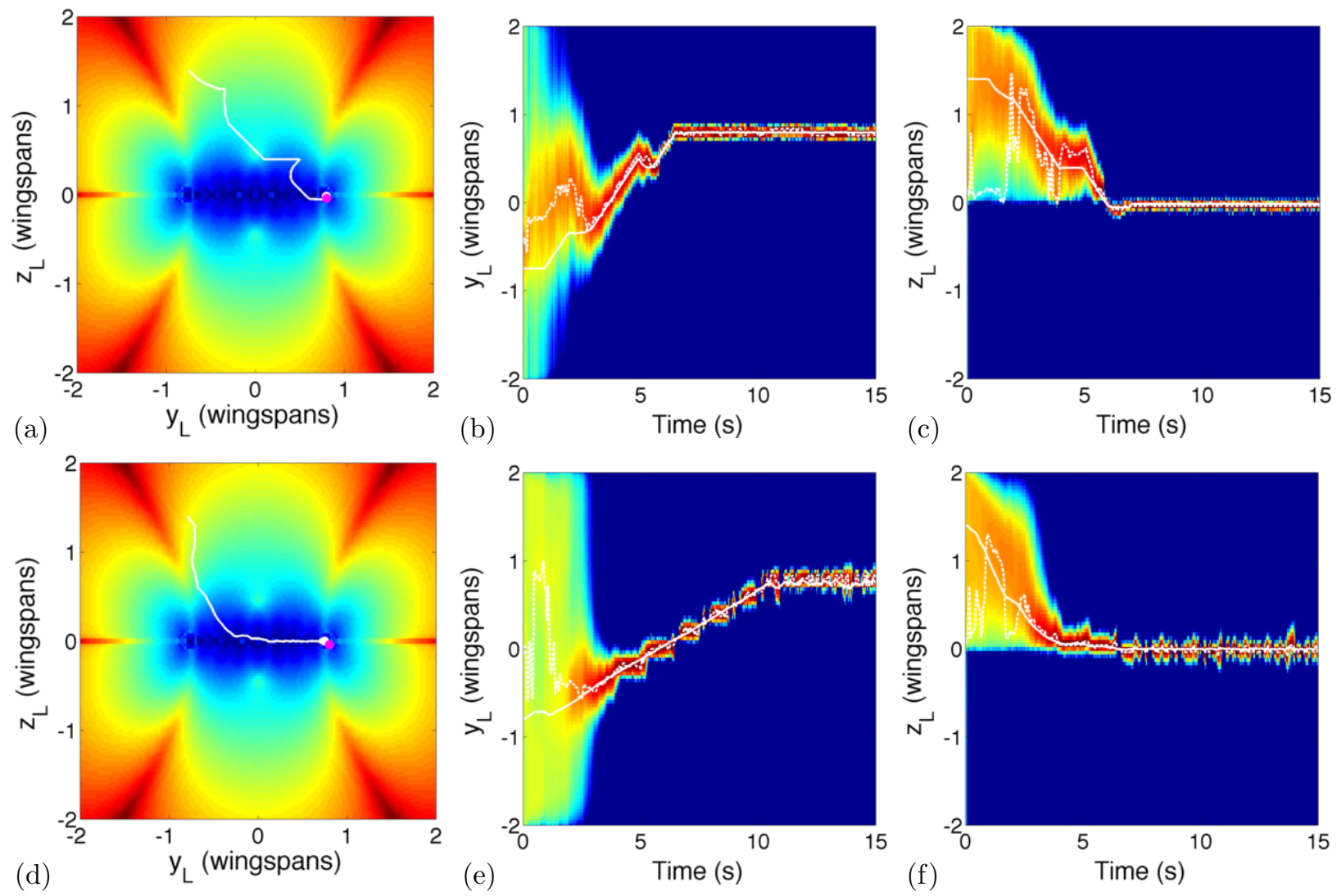

Figure 5. Simulation illustrating formation flight with optimal control algorithms of Section III. (a) Leader aircraft position trajectory using receding-horizon control; (b,c) marginal probability densities for relative position over time. (d-f) Illustration of wavefront-propagation control using estimated relative position.

\section{B. Autonomous Aerial Refueling}

In aerial refueling the follower aircraft intercepts a filling nozzle that extends outward from the tail of the leader ${ }^{1}$ by maintaining a desired relative position of $\mathbf{r}_{\text {des }}=(0,0)$ wingspans. This implementation assumes the aerodynamic effects of the filling nozzle on the follower aircraft are minimal at the sensor positions. Figure 6 illustrates simulation results with the same initial and operating conditions described in Section IV.A.

Figures 6(a,d) show the trajectories of the leader aircraft (white) plotted over the unobservability index for the receding-horizon and wavefront-propagation control algorithms, respectively. Figures $6(\mathrm{~b}, \mathrm{c})$ show the marginal probability densities of the relative position estimates plotted versus time for the receding-horizon method, whereas Figures 6(e,f) show the marginal probability densities resulting from the wavefront-propagation control. Both control algorithms steer the leader to the desired position $\mathbf{r}_{d e s}$, but the receding-horizon method has less control authority in the $y_{L}$ direction due to the estimation error. Initial errors in the position cause the wavefront-propagation control to steer the follower aircraft to the desired position with slight deviations toward unobservable regions. 

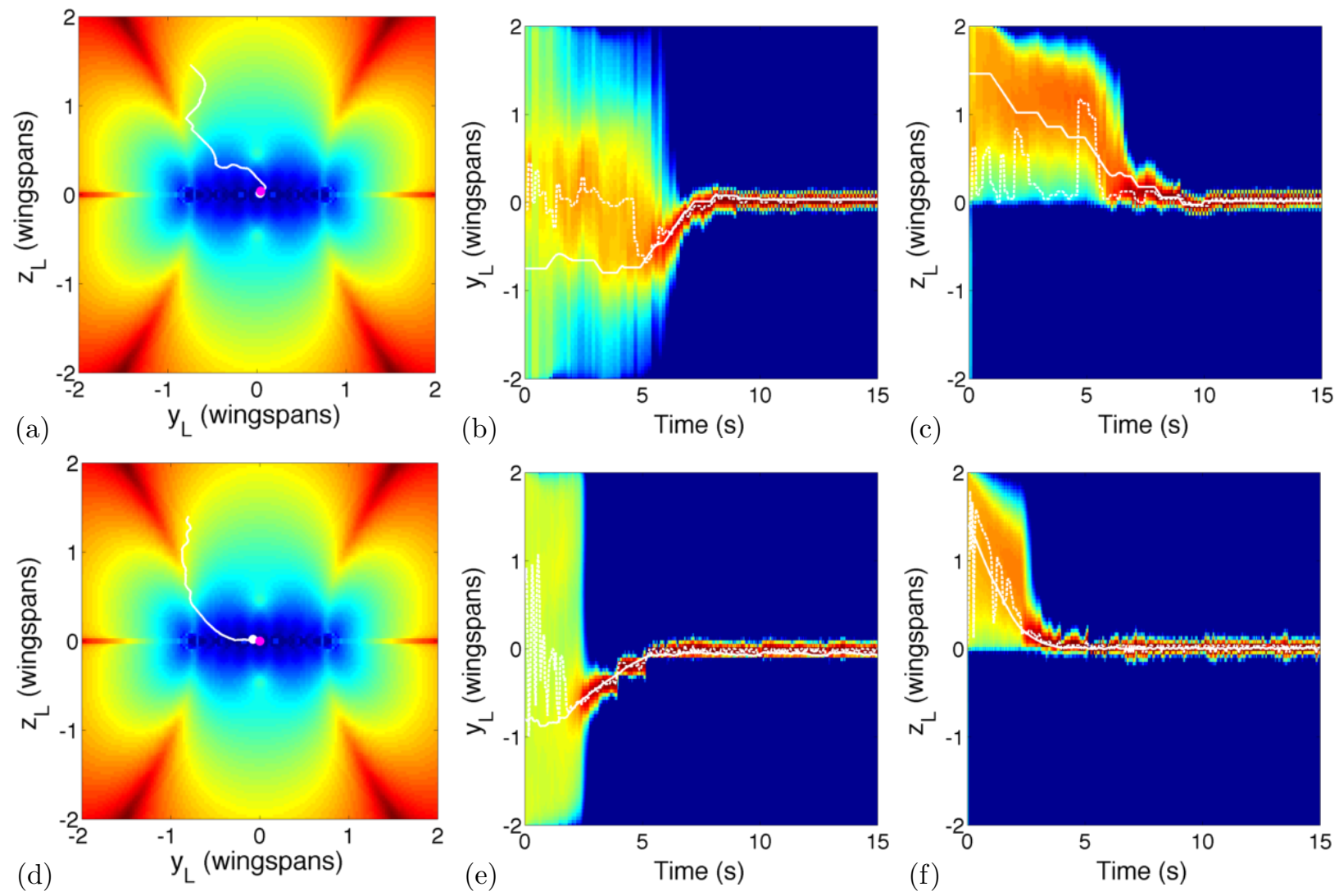

Figure 6. Simulation illustrating aerial refuel positioning with optimal control algorithms of Section III. (a)-(c) Leader aircraft position trajectory and marginal probability densities using receding-horizon method. (d)-(f) Trajectory and marginal probability densities using wavefront-propagation method.

\section{Conclusion}

Aircraft may operate in close proximity to increase flight endurance, particularly for aerial refueling and reducing induced drag. This paper uses lifting-line theory to model a two-aircraft formation and derives observability-based optimal control strategies that steer the follower aircraft to a desired relative position. The follower aircraft collects noisy measurements of the aerodynamic signature created by the leader's wake. The observability of the leader aircraft is assessed using measures of the empirical observability gramian and a recursive Bayesian filter is implemented to estimate the leader's wake parameters. Recedinghorizon and wavefront-propagation optimal control algorithms use the unobservability index as a cost metric and incorporate estimates of the leader's state provided by the Bayesian filter. Numerical simulations of formation flight and aerial refueling applications illustrate that the proposed control algorithms successfully steer the vehicle to a desired relative position while simultaneously estimating the wake parameters. 


\section{Acknowledgments}

The authors would like to acknowledge Mazier Hemati for discussions related to this work. This work is supported by the Office of Naval Research under Grant No. N00014-12-1-0149 and the Air Force Office of Scientific Research under Grant No. FA95501310162.

\section{References}

${ }^{1}$ Dogan, A., Sato, S., and Blake, W., "Flight Control and Simulation for Aerial Refueling," In Proc. of AIAA Guidance, Navigation, and Control Conf., San Francisco, CA, August 2005.

${ }^{2}$ Dogan, A., Lewis, T. A., and Blake, W., "Flight Data Analysis and Simulation of Wind Effects During Aerial Refueling," J. of Aircraft, Vol. 45, No. 6, Nov.-Dec. 2008, pp. 2036-2048.

${ }^{3}$ Blake, W. and Multhopp, D., "Design, Performance, and Modeling Considerations for Close Formation Flight," In Proc. of the AIAA Guidance, Navigation, and Control Conf., August 1998.

${ }^{4}$ Maskew, B., "Formation Flying Benefits Based on Vortex Lattice Calculations," Tech. rep., NASA CR-151974, 1974.

${ }^{5}$ Kent, T. E. and Richards, A. G., "A Geometric Approach to Optimal Routing for Commercial Formation Flight," In Proc. of AIAA Guidance, Navigation, and Control Conf., Minneapolis, MN, 2012.

${ }^{6}$ DeVries, L. and Paley, D. A., "Multi-vehicle Control in a Strong Flowfield with Application to Hurricane Sampling," AIAA J. of Guidance, Control, and Dynamics, Vol. 35, No. 3, 2012, pp. 794-806.

${ }^{7}$ Dunbabin, M. and Marques, L., "Robotics for Environmental Monitoring," IEEE Robotics and Automation Magazine, February 2012, pp. 24-39.

${ }^{8}$ Sydney, N. and Paley, D. A., "Multi-vehicle Control and Optimization for Spatiotemporal Sampling," In Proc. of the IEEE Conf. on Decision and Control, December 2011, pp. 5607-5612.

${ }^{9}$ Hummel, D., "Aerodynamic Aspects of Formation Flight in Birds," J. of Theoretical Biology, Vol. 104, No. 3, 1983, pp. 321-347.

${ }^{10}$ Ning, S. A., Aircraft Drag Reduction Through Extended Formation Flight, Ph.D. thesis, Stanford University, August 2011.

${ }^{11}$ Chichka, D. F., Wolfe, J. D., and Speyer, J. L., "Aerodynamically Coupled Formation Flight of Aircraft," In Proc. of the 10th Med. Conf. on Control and Automation, Lisbon, Portugal, July 2002.

${ }^{12}$ Thien, H. P., Moelyadi, M. A., and Muhammad, H., "Effects of Leader's Position and Shape on Aerodynamic Performances of V Flight Formation," CoRR, Vol. abs/0804.3879, 2008.

${ }^{13}$ Pachter, M., D’Azzo, J. J., and Proud, A. W., "Tight Formation Flight Control," AIAA J. of Guidance, Control, and Dynamics, Vol. 24, No. 2, March-April 2001, pp. 246-254.

${ }^{14}$ Ding, J. and Fan, Q., "A Multi-UAV Tight Formation Flight Controller," 2012 IEEE Int. Conf. on Comp. Science and Automation Eng. (CSAE), Vol. 1, May 2012, pp. 60-64.

${ }^{15}$ Bangash, Z. A., Sanchez, R. P., and Ahmed, A., "Aerodynamics of Formation Flight," In Proc. of the AIAA Aerospace Sciences Meeting, AIAA 2004-725, Reno, NV, January 2004.

${ }^{16} \mathrm{Katz}$, J. and Plotkin, A., Low-Speed Aerodynamics, Cambridge University Press, 2nd ed., 2001.

${ }^{17}$ Moran, J., An Introduction to Theoretical and Computational Aerodynamics, John Wiley and Sons, 1984 . 
${ }^{18}$ Hemati, M. S., Eldredge, J. D., and Speyer, J. L., "Wake Sensing for Aircraft Formation Flight," In Proc. of AIAA Guidance, Navigation, and Control Conf., Minneapolis, MN, August 2012.

${ }^{19}$ Benson, D. A., A Gauss Pseudospectral Transcription for Optimal Control, Ph.D. thesis, Massachusetts Institute of Technology, November 2004.

${ }^{20}$ Sethian, J. A., Level set methods and fast marching methods: evolving interfaces in computational geometry, fluid mechanics, computer vision, and materials science, Cambridge University Press, 1999.

${ }^{21}$ Hermann, R. and Krener, A. J., "Nonlinear Controllability and Observability," IEEE Trans. Auto. Control, Vol. 22, No. 5, 1977, pp. 728-740.

${ }^{22}$ Lall, S., Marsden, J., and Glavaski, S., "A Subspace Approach to Balanced Truncation for Model Reduction of Nonlinear Control Systems," Int. J. of Robust and Nonlinear Cont., Vol. 12, No. 6, 2002, pp. 519-535.

${ }^{23}$ Krener, A. and Ide, K., "Measures of Unobservability," Proc. 48th IEEE Conf. Decision and Cont., Shanghai, China, Dec. 2009, pp. 6401-6406.

${ }^{24}$ Batista, P., Silvestre, C., and Oliveira, P., "Single Range Aided Navigation and Source Localization: Observability and Filter Design," Systems and Control Letters, Vol. 60, No. 8, 2011, pp. 665 - 673.

${ }^{25}$ Wouwer, A. V., Point, N., Porteman, S., and Remy, M., "An Approach to the Selection of Optimal Sensor Locations in Distributed Parameter Systems," J. of Proc. Cont., Vol. 10, No. 4, Aug. 2000, pp. 291300 .

${ }^{26}$ Singh, A. K. and Hahn, J., "Determining Optimal Sensor Locations for State and Parameter Estimation for Stable Nonlinear Systems," Industrial and Eng. Chem. Research, Vol. 44, No. 15, June 2005, pp. 5645-5659.

${ }^{27}$ DeVries, L., Majumdar, S. J., and Paley, D. A., "Observability-based Optimization of Coordinated Sampling Trajectories for Recursive Estimation of a Strong, Spatially Varying Flowfield," J. of Intelligent and Robotic Systems, Vol. 70, No. 1-4, April 2013, pp. 527-544.

${ }^{28}$ Hinson, B. T., Binder, M. K., and Morgansen, K. A., "Path planning to optimize observability in a planar uniform flow field," Proc. American Control Conf. (ACC), 2013, pp. 1394-1401.

${ }^{29}$ Skogestad, S. and Postlethwaite, I., Multivariable Feedback Control: Analysis and Design, Wiley and Sons, West Sussex, England, 1996.

${ }^{30}$ Hinson, B. T. and Morgansen, K. A., "Flowfield Estimation in the Wake of a Pitching and Heaving Airfoil," In Proc. of the American Cont. Conf., June 2012.

${ }^{31}$ Hahn, J. and Edgar, T. F., "A Gramian Based Approach to Nonlinear Quantification and Model Classification," Industrial and Engineering Chemistry Research, Vol. 40, 2001, pp. 5724-5731.

${ }^{32}$ Bergman, N., Recursive Bayesian Estimation Navigation and Tracking Applications, Ph.D. thesis, Department of Electrical Engineering, Linkoping University, Sweden, 1999.

${ }^{33}$ Bar-Shalom, Y., Rong Li, X., and Kirubarajan, T., Estimation with Applications to Tracking and Navigation: Theory Algorithms and Software, Wiley-Interscience, 1st ed., June 2001.

${ }^{34}$ Huntington, G. T., Advancement and Analysis of a Gauss Pseudospectral Transcription for Optimal Control, Ph.D. thesis, Massachusetts Institute of Technology, May 2007. 